Check for updates

Cite this: Mater. Chem. Front., 2020, 4, 1648

Received 28th February 2020

Accepted 10th April 2020

DOI: 10.1039/d0qm00116c

rsc.li/frontiers-materials

\title{
Highly efficient exciplex-based OLEDs incorporating a novel electron donor
}

\author{
Qi-Sheng Tian, ${ }^{a}$ Xiang-Dong Zhu ${ }^{a}$ and Liang-Sheng Liao (D) *ab
}

\begin{abstract}
A novel triphenylamine (TPA) derivative molecule 4,4,8,8,12,12-hexaphenyl-8,12-dihydro-4H-benzo[9,1]quinolizino[3,4,5,6,7-defg]acridine (DEX) is presented as an electron donor to form an effective exciplex system with an electron acceptor molecule ((1,3,5-triazine-2,4,6-triyl)tris(benzene-3,1-diyl))tris(diphenylphosphine oxide) (PO-T2T) for fabricating high performance exciplex-based organic light-emitting diodes (OLEDs). If the DEX:PO-T2T exciplex itself is used as an emitting layer without any doped luminescent emitter in an OLED, it can exhibit green emission with a maximum power efficiency $\left(P E_{\max }\right)$, current efficiency $\left(C E_{\max }\right)$, and external quantum efficiency $\left(E Q E_{\max }\right)$ of $44.6 \mathrm{~lm} \mathrm{~W}^{-1}, 36.0 \mathrm{~cd} \mathrm{~A}$, and $11.2 \%$, respectively. The high device performance indicates that the green exciplex would have balanced charge transport properties, a broad recombination zone, and a suitable triplet energy level $\left(E_{T}\right)$, which is therefore expected to serve as an ideal host for a red phosphorescent emitter. Indeed, due to the desirable thermal and electrical properties of donor DEX, an optimized exciplex-based red phosphorescent OLED (PhOLED), doped with iridium(III) bis(2-methyldibenzo[f,h]quinoxaline)acetylacetonate (Ir(MDQ) ${ }_{\mathbf{2}}($ acac)), achieved an impressive $P E_{\max }, C E_{\max }$, and $E E_{\text {max }}$ of $46.1 \mathrm{~lm} \mathrm{~W}^{-1}, 36.0 \mathrm{~cd} \mathrm{~A}^{-1}$, and $24.5 \%$ as well as a low operating voltage (2.4 $\mathrm{V}$ at $0.2 \mathrm{~mA} \mathrm{~cm}^{-2}$ ). Remarkably, both an excellent EQE over $22 \%$ and low operating voltage of $3.4 \mathrm{~V}$ remained even at a high brightness of $1000 \mathrm{~cd} \mathrm{~m}^{-2}$. The high EQE and low efficiency roll-off reveal that the new exciplex system using novel donor DEX enables improved OLED performance, which paves the way to practical applications of OLEDs.
\end{abstract}

\section{Introduction}

Organic light-emitting diodes (OLEDs) have opened a reliable avenue in optoelectronic devices due to their inherent characteristics, such as high-contrast, high-efficiency, and flexibility. ${ }^{1-3}$ The development of conventional fluorescent material-based OLEDs is impeded by the limited internal quantum efficiency (IQE) of $25 \%$ without being capable of utilization of the remaining $75 \%$ noraditive triplet excitons. ${ }^{4}$ Over the past decades, 2nd generation OLEDs employing phosphorescent emitters have offered striking EQE via harvesting both singlet and triplet excitons for $100 \%$ internal quantum efficiency (IQE). ${ }^{5-7}$ Recently, thermally activated delayed fluorescence (TADF) materials commonly exhibit typical intramolecule charge transfer (ICT) behavior, which show an effective reverse intersystem crossing (RISC) process and small singlet-triplet energy splitting $\left(\Delta E_{\mathrm{ST}}\right) \cdot{ }^{8-10}$ So, they have the ability to efficiently

\footnotetext{
${ }^{a}$ Jiangsu Key Laboratory for Carbon-Based Functional Materials \& Devices, Institute of Functional Nano \& Soft Materials (FUNSOM), Soochow University, Suzhou, Jiangsu 215123, China. E-mail: lsliao@suda.edu.cn

${ }^{b}$ Institute of Organic Optoelectronics, Jiangsu Industrial Technology Research Institute (JITRI), Wujiang, Suzhou, Jiangsu 215211, China

$\dagger$ Electronic supplementary information (ESI) available. See DOI: 10.1039/ d0qm00116c
}

utilize both singlet and triplet excitons by converting triplet excitons from the triplet excited state $\left(\mathrm{T}_{1}\right)$ into the singlet excited state $\left(S_{1}\right)$. Thereby, phosphorescent and TADF materials are widely considered as promising strategies to sufficiently utilize excitons for achieving high performance devices.

Exciplex systems comprising an electron donor and an electron acceptor can contribute to intermolecular charge transfer accompanied by effective RISC and a small $\Delta E_{\mathrm{ST}}{ }^{11-13}$ So the exciplex is considered as another type of efficient TADF emitter to fabricate high-efficiency exciplex-based fluorescent OLEDs. ${ }^{13,14}$ It's worth noting that an exciplex is generally a combination of an electron-transporting material and a holetransporting material, which can realize balanced charge transport, a negligible injection barrier, and a broad recombination zone. Therefore, several exciplex forming hosts have been developed and they played a crucial role to reduce the operating voltage in devices for enhancing the power efficiency (PE). ${ }^{15-17}$ Ideally, an exciplex system can be not only regarded as the emitting layer to fabricate efficient fluorescent OLEDs, but also serve as a co-host to enhance the performance of emitters. Thus, designing an effective exciplex system is in demand for the development of OLEDs.

However, it is relatively rare to select an electron donor for fabricating an exciplex system in order to achieve high 
performance OLEDs, because most researchers pay more attention to designing electron acceptor materials, e.g., star-shaped 1,3,5-triazine/cyano hybrid molecule CN-T2T, ${ }^{18}$ heptazine derivative $\mathrm{HAP}-3 \mathrm{MF},{ }^{13}$ benzimidazole-triazine-based electron acceptor PIM-TRZ, ${ }^{19}$ and isomeric N-linked benzoimidazole materials (iTPyBIB, iTPBIPy, and iTPyBIPy). ${ }^{20}$ These electron acceptor materials have shown significant roles in highly efficient exciplex systems. In contrast, the common electron donors widely utilized in exciplex systems are mainly restricted to commercial materials, such as 1,3-di-9-carbazolylbenzene (mCP) ${ }^{15}$ 1,1-bis[4-[N' $N^{\prime}, N^{\prime}$-di(p-tolyl)amino $]$-phenyl $]$ cyclohexane (TAPC), ${ }^{17} 4,4^{\prime}, 4^{\prime \prime}$-tris[3-methylphenyl(phenyl)amino] triphenylamine ( $m$-MTDATA), ${ }^{11}$ and $N, N, N$-tris(4-(9-carbazolyl)phenyl)amine (TCTA). ${ }^{21}$ It is therefore necessary to develop more appropriate electron donor materials to match suitable electron acceptor materials in fabricating effective exciplex systems for obtaining high performance OLEDs with high EQE and PE, and low operating voltage. With the expectation to design ideal electron donor materials, the following prerequisites should be mentioned: (i) obtaining a suitable $E_{\mathrm{T}}$ of the donor for ensuring effective energy transfer; (ii) achieving satisfactory highest occupied molecular orbital (HOMO) and lowest unoccupied molecular orbital (LUMO) levels to match well with the acceptor material and the adjacent charge transport materials for ensuring the formation of an exciplex; and (iii) realizing low cost, an uncomplicated synthesis procedure, and good thermal properties.

Reported herein is a new triphenylamine (TPA) derivativebased electron donor molecule 4,4,8,8,12,12-hexaphenyl-8,12dihydro-4H-benzo[9,1]quinolizino[3,4,5,6,7-defg]acridine (DEX) that shows a desirable triplet energy level $\left(E_{\mathrm{T}}=2.75 \mathrm{eV}\right)$, good thermal stability, a facile synthetic process, and appropriate HOMO and LUMO levels. The electron donor DEX and an electron-transporting molecule ((1,3,5-triazine-2,4,6-triyl)tris(benzene-3,1-diyl))tris(diphenylphosphine oxide) (PO-T2T) were selected to fabricate a suitable exciplex system. An exciplexbased fluorescent OLED was first proposed and it can exhibit green emission peaking at $520 \mathrm{~nm}$. Importantly, the green exciplex-based OLED can realize a maximum EQE (EQE $\left.E_{\max }\right)$ and $\mathrm{PE}\left(\mathrm{PE}_{\max }\right)$ and low operating voltage (at $0.2 \mathrm{~mA} \mathrm{~cm} \mathrm{~cm}^{-2}$ ) of $11.2 \%, 48.1 \mathrm{~lm} \mathrm{~W}^{-1}$ and $2.5 \mathrm{~V}$, respectively, which are superior to those of traditional fluorescent OLEDs. Furthermore, the green emission exciplex was proved as a promising co-host in devices to reduce the operating voltage and to broaden the exciton recombination zone for improving the performance of red phosphorescent emitters, such as iridium(III) bis(2-methyldibenzo $[f, h]$ quinoxaline)acetylacetonate $\operatorname{Ir}(\mathbf{M D Q})_{2}$ (acac). Finally, benefiting from the exciplex forming co-host, the optimized red phosphorescent OLED (PhOLED) with $\operatorname{Ir}(\mathbf{M D Q})_{2}$ (acac) as the emitter shows remarkable performance with an $\mathrm{EQE}_{\max }, \mathrm{PE}_{\max }$, and operating voltage (at $0.2 \mathrm{~mA} \mathrm{~cm}^{-2}$ ) of $24.5 \%, 46.1 \mathrm{~lm} \mathrm{~W}^{-1}$ and $2.4 \mathrm{~V}$, respectively. These superior results of the efficient green and red exciplex-based OLEDs can be ascribed to the properties of DEX, which has a suitable $E_{\mathrm{T}}$, good photophysical characteristics, better carrier transport capability, and suitable frontier molecular orbitals (FOMs) as well as a reasonable device structure to facilitate charge injection and recombination.

\section{Results and discussion}

The synthetic route and chemical structure of the donor DEX are outlined in Scheme 1. The specific steps of the synthetic process are expressed in the Experimental section. It clearly shows that the synthetic process is particularly uncomplicated and convenient, proving that it has the practical potential for fabrication and applications. For investigating the electronic structure of DEX, density functional theory (DFT) calculations were applied at the B3LYP/6-31G(d) level. The HOMO/LUMO FMO distributions and energy levels are depicted in Fig. 1. The HOMO energy level $(-5.03 \mathrm{eV})$ and LUMO energy level $(-2.03 \mathrm{eV})$ of DEX can be calculated. From the ground state geometry, a singlet energy level $\left(E_{\mathrm{S}}\right)$ and $E_{\mathrm{T}}$ of 3.1 and $2.6 \mathrm{eV}$ can be found, respectively.

To explore the electrochemical properties of DEX, the HOMO energy level was estimated through ultraviolet photospectroscopy (UPS) measurements (Fig. 2a). The UV-vis absorption and photoluminescence (PL) spectra of DEX in dilute $\mathrm{CH}_{2} \mathrm{Cl}_{2}$ solution at room temperature are shown in Fig. $2 \mathrm{~b}$. From the UPS curves we can conclude that DEX has a HOMO energy level of $-5.8 \mathrm{eV}$, which is helpful to transfer holes. ${ }^{22}$ The maximum absorption at a wavelength around $300 \mathrm{~nm}$ is ascribed mainly to the $\pi-\pi^{*}$ intramolecular transition. The onset of the UV-vis absorption edge of DEX is at about $344 \mathrm{~nm}$, so the optical bandgap $\left(E_{\mathrm{g}}\right)$ can be estimated to be $3.6 \mathrm{eV}$. Hence, the LUMO energy level of DEX can be calculated according to the equation $E_{\mathrm{g}}=-\left(E_{\text {HOMO }}-E_{\mathrm{LUMO}}\right)$ as $c a$. $-2.2 \mathrm{eV}$. Meanwhile, the electrochemical properties of DEX were investigated by cyclic voltammetry (CV); from the reversible oxidation curve we can conclude that DEX is electrochemically

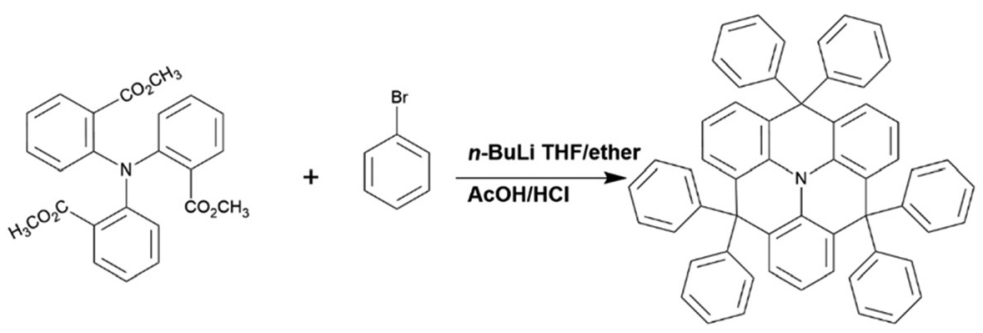

Scheme 1 Synthetic route and chemical structure of DEX. 


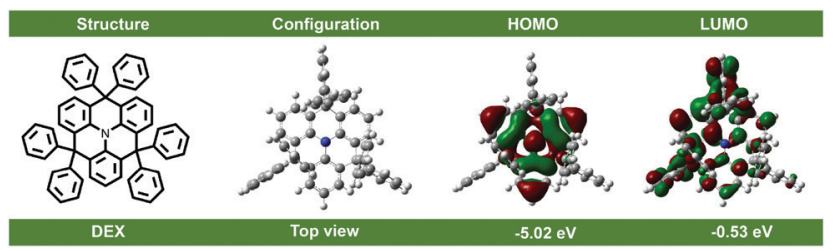

Fig. 1 Chemical structure, calculated molecular HOMO and LUMO distributions, and energy levels of DEX.
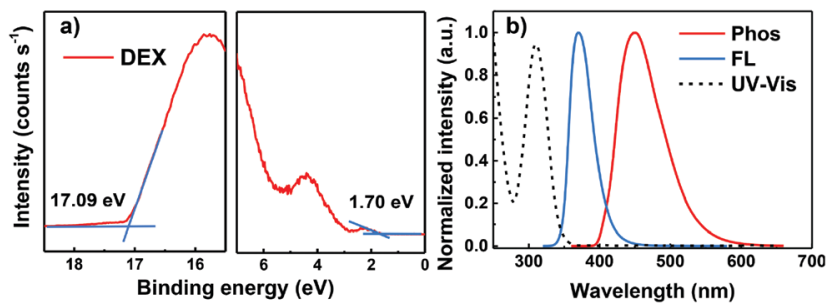

Fig. 2 (a) UPS spectrum of the DEX film, and (b) UV-vis absorption in $\mathrm{CH}_{2} \mathrm{Cl}_{2}$ and the fluorescence spectrum in toluene $(300 \mathrm{~K})$ as well as the phosphorescence (toluene, 77K) spectrum of DEX.

stable (shown in Fig. S4, ESI $\dagger$ ). The wavelength of the peak emission of the phosphorescence spectrum at $77 \mathrm{~K}$ is $450 \mathrm{~nm}$, indicating that the $E_{\mathrm{T}}$ of DEX is about $2.75 \mathrm{eV}$. The high $\mathrm{T}_{1}$ level of DEX is beneficial to ensure energy transfer from the host to the emitters effectively. ${ }^{23}$ The device performance is susceptible to the thermal properties of the organic material. As shown in Fig. S3 (ESI $\dagger$ ), DEX exhibits distinguished thermal stability with a high decomposition temperature $\left(T_{\mathrm{d}}\right)$ of $397{ }^{\circ} \mathrm{C}$ (refers to $5 \%$ weight loss), which was calculated from thermogravimetric analysis (TGA). The high $T_{\mathrm{d}}$ indicates that DEX has excellent thermal properties, which is ascribed to its rigid molecular structure. The detailed photophysical properties are summarized in Table 1.

In order to fabricate an exciplex system with an effective intermolecular charge transfer process, a considerable offset $(>0.4 \mathrm{eV})$ between the HOMO/LUMO energy levels of the donor/acceptor should be achieved. ${ }^{24}$ Hence, an electrontransporting material PO-T2T was chosen to form an exciplex with DEX owing to its ideal HOMO/LUMO $(-3.5 /-7.5 \mathrm{eV})$ energy levels. ${ }^{15}$ Thus the energy level offset between DEX and PO-T2T is large enough to form an exciplex. The molecular configurations of DEX and PO-T2T are depicted in Fig. 3a. The exciplex forming process and energy level diagrams are illustrated in Fig. $3 \mathrm{~b}$.

Table 1 Summary of the photophysical properties of DEX

\begin{tabular}{llllllll}
\hline Molecule & $\begin{array}{l}\mathrm{Abs} \lambda_{\max }{ }^{a} \\
{[\mathrm{~nm}]}\end{array}$ & $\begin{array}{l}\mathrm{PL} \lambda_{\max }{ }^{a} \\
{[\mathrm{~nm}]}\end{array}$ & $\begin{array}{l}T_{\mathrm{d}}{ }^{b} \\
{\left[{ }^{\circ} \mathrm{C}\right]}\end{array}$ & $\begin{array}{l}E_{\mathrm{g}}{ }^{c} \\
{[\mathrm{eV}]}\end{array}$ & $\begin{array}{l}E_{\mathrm{T}}{ }^{d} \\
{[\mathrm{eV}]}\end{array}$ & $\begin{array}{l}\mathrm{HOMO}^{e} \\
{[\mathrm{eV}]}\end{array}$ & $\begin{array}{l}\mathrm{LUMO}^{f} \\
{[\mathrm{eV}]}\end{array}$ \\
\hline DEX & 344 & 370 & 397 & 3.6 & 2.7 & -5.8 & -2.2
\end{tabular}

${ }^{a}$ Tested in toluene solution at room temperature. ${ }^{b} T_{\mathrm{d}}$ : Decomposition temperature. ${ }^{c} E_{\mathrm{g}}$ : Optical band gap energy was measured from the corresponding absorption onset. ${ }^{d} E_{\mathrm{T}}$ : Measured in a toluene glass matrix at $77 \mathrm{~K} .{ }^{e}$ HOMO energy level was calculated from UPS data. ${ }^{f}$ LUMO level was obtained from the results of the HOMO and $E_{\mathrm{g}}$.
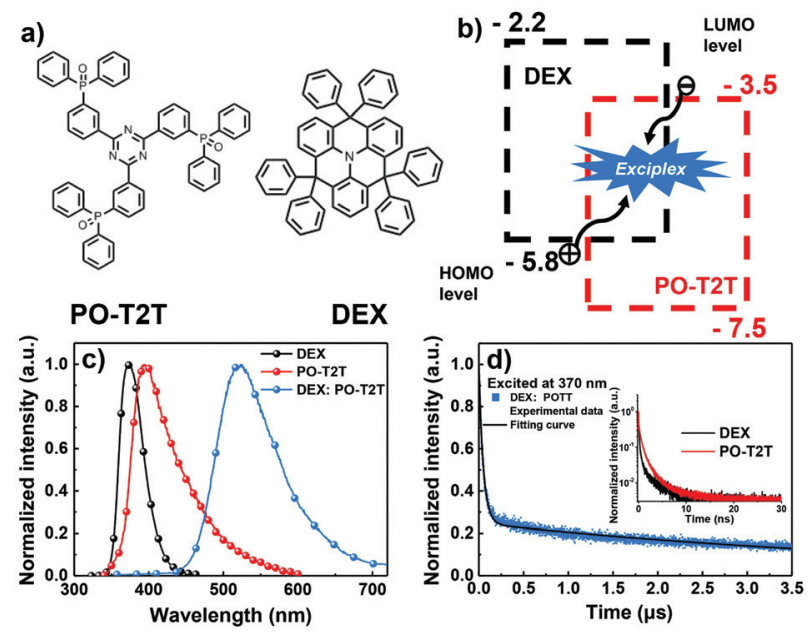

Fig. 3 (a) Molecular structures of PO-T2T and DEX, (b) the process of exciplex formation of DEX:PO-T2T with the HOMO and LUMO energy levels, (c) the PL spectra of DEX, PO-T2T, and DEX:PO-T2T films at $300 \mathrm{~K}$, and (d) PL decay curves of exciplex DEX:PO-T2T and the pristine materials at $300 \mathrm{~K}$.

To verify the formation of the DEX:PO-T2T exciplex, the PL spectrum of exciplex DEX:PO-T2T with peak emission at a wavelength of $520 \mathrm{~nm}$ was observed (shown in Fig. 3c). It is clearly found that the PL of the exciplex is red shifted compared with those of DEX ( $370 \mathrm{~nm}$ ) and PO-T2T ( $410 \mathrm{~nm}$ ), which means that an exciplex was generated. ${ }^{25}$ The photo energy level of exciplex $\left(E_{\mathrm{S}, \mathrm{ex}}\right)$ DEX:PO-T2T was experimentally measured to be $2.38 \mathrm{eV}$ according to the PL peak emission $(520 \mathrm{~nm})$, which is consistent with the energy difference between the HOMO energy level $(-5.8 \mathrm{eV})$ of DEX and the LUMO energy level $(-3.5 \mathrm{eV})$ of PO-T2T. ${ }^{23}$ The triplet energy level of the exciplex $\left(E_{\mathrm{T}, \mathrm{ex}}\right)$ was estimated to be $2.3 \mathrm{eV}$ based on previously reported exciplex-based work, which means that the exciplex system has a dramatically negligible $\Delta E_{\mathrm{ST}} \cdot{ }^{26-28}$ These results demonstrate that electron-hole coupling was generated under external excitation. Additionally, the transient fluorescence decay curves of DEX:PO-T2T and the pristine molecules are shown in Fig. 3d. DEX and PO-T2T only exhibit decay lifetimes of a few nanoseconds. In contrast, prompt and delayed components could be observed in the DEX:PO-T2T transient decay curve. The exciplex exhibits a short and long decay lifetime of $30 \mathrm{~ns}$ and $1.2 \mu \mathrm{s}$, respectively. The aforementioned results reveal certain evidence that the formation of exciplex DEX:PO-T2T possessing small $\Delta E_{\mathrm{ST}}$ with an effective RISC process is achieved.

To further study the potential advantages of the DEX:PO-T2T exiplex system, a DEX:PO-T2T exciplex-based OLED was first fabricated to investigate its electroluminescence (EL) properties. For better comparison, a classic hole-transporting material 4,4,4-tris $\left(N\right.$-carbazolyl)triphenylamine (TCTA) $\left(E_{\mathrm{T}}=2.76\right)$, which has HOMO and LUMO levels of -5.8 and $-2.4 \mathrm{eV}$, respectively, was employed to fabricate an exciplex with PO-T2T. ${ }^{29}$ As shown in Fig. S5 (ESI $\dagger$ ), the exciplex TCTA:PO-T2T shows a broad spectrum with a wavelength of $520 \mathrm{~nm}$, which is red shifted compared with TCTA $(400 \mathrm{~nm})$ and PO-T2T, indicating the 
formation of an exciplex. The $E_{\mathrm{T}}$ values of exciplexes DEX:PO-T2T $(2.3 \mathrm{eV})$ and TCTA:PO-T2T are very close according to their emission peaks $(520 \mathrm{~nm})$. The devices were designed with the structure of ITO/HAT-CN $(10 \mathrm{~nm}) /$ TAPC $(30 \mathrm{~nm}) / \mathbf{D E X}$ or TCTA (10 nm)/DEX:PO-T2T or TCTA:PO-T2T $(20 \mathrm{~nm}) /$ PO-T2T $(45 \mathrm{~nm}) /$ Liq $(2 \mathrm{~nm}) / \mathrm{Al}(120 \mathrm{~nm})$. Hexaaza-triphenylene hexacarbonitrile (HAT-CN) and lithium quinolate (Liq) were employed as hole and electron-injecting layers (HIL and EIL), respectively. ${ }^{25}$ TAPC can serve as a hole-transporting layer (HTL) owing to its suitable HOMO energy level $(-5.6 \mathrm{eV})$ and high $E_{\mathrm{T}}(2.98 \mathrm{eV}),{ }^{30}$ which matches well with DEX and TCTA, so that the holes can smoothly transfer from the anode to the emission zone for generating excitons with electrons. The additional $10 \mathrm{~nm}$ DEX or TCTA was introduced as an electron-blocking layer to prevent the undesirable formation of exciplex TAPC:PO-T2T. ${ }^{31}$

The EL characteristics of the exciplex DEX:PO-T2T $\left(\right.$ Green $\left._{1}\right)$ and TCTA:PO-T2T $\left(\right.$ Green $\left._{2}\right)$ OLEDs are presented in Fig. 4 . The exciplex-based OLEDs realized typical green emission with a peak at $520 \mathrm{~nm}$, which is consistent with the PL one. So all excitons generated on DEX:PO-T2T and TCTA:PO-T2T are sufficiently used for the emission of the exciplex without energy loss. As the current density-voltage-luminance $(J-V-L)$ curves show (Fig. 4a), low operating voltages of 2.6 and $3.5 \mathrm{~V}$ at a luminance of 100 and $1000 \mathrm{~cd} \mathrm{~m}^{-2}$ were achieved in Green ${ }_{1}$. Notably, these values are lower than that of the TCTA-based OLED, indicating that DEX has better carrier transport properties compared with TCTA. ${ }^{32}$ The low operating voltages of both devices are due to the good injection and the direct recombination of holes from the HOMO of DEX or TCTA and electrons from the LUMO of PO-T2T. Because of the low operating voltages of the DEX-based device, an excellent $\mathrm{PE}_{\max }$ and maximum $\mathrm{CE}\left(\mathrm{CE}_{\max }\right)$ of $44.1 \mathrm{~lm} \mathrm{~W}^{-1}$ and $36 \mathrm{~cd} \mathrm{~A}^{-1}$ were achieved. For the TCTA-based OLED, a $\mathrm{PE}_{\max }$ and $\mathrm{CE}_{\max }$ of $17.4 \mathrm{~lm} \mathrm{~W}^{-1}$ and $15.0 \mathrm{~cd} \mathrm{~A}^{-1}$ can be realized. Finally, the DEX and TCTA-based devices exhibited an $\mathrm{EQE}_{\max }$ of $11.2 \%$ and $4.0 \%$, respectively. The substantial difference in the performance between the two devices is mainly ascribed to the superior properties of DEX, having good carrier transport and

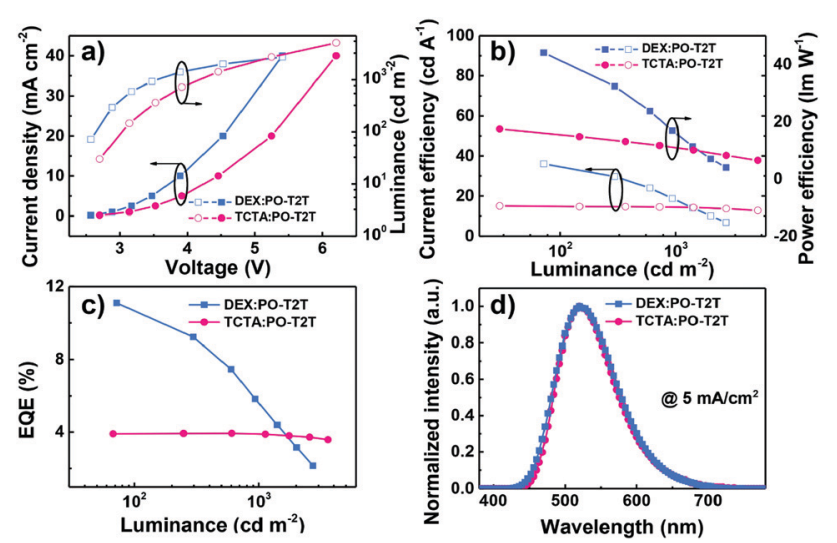

Fig. 4 (a) $J-V-L$ plots, (b) CE-luminance-PE plots, (c) EQE-luminance plots, and (d) EL (at $5 \mathrm{~mA} \mathrm{~cm}^{-2}$ ) spectra of DEX:PO-T2T and TCTA:PO-T2T exciplex-based devices.
Table 2 Summary of the EL performance for green and red OLEDs

\begin{tabular}{llllll}
\hline Devices $^{a}$ & $V^{b}[\mathrm{~V}]$ & $\eta_{\mathrm{CE}}{ }^{c}\left[\mathrm{~cd} \mathrm{~A}^{-1}\right]$ & $\eta_{\mathrm{PE}}{ }^{c}\left[\mathrm{~lm} \mathrm{~W}^{-1}\right]$ & $\eta_{\mathrm{ext}}{ }^{c}[\%]$ & $\mathrm{CIE}^{d}[x, y]$ \\
\hline Green $_{1}$ & 2.5 & $36.0,18.2$ & $44.1,16.8$ & $11.2,5.8$ & $0.29,0.55$ \\
Green $_{2}$ & 2.7 & $15.0,14.3$ & $17.5,11.4$ & $4.0,3.8$ & $0.29,0.55$ \\
$\operatorname{Red}_{1}$ & 2.7 & $39.0,31.5$ & $45.3,24.2$ & $19.7,16.0$ & $0.54,0.42$ \\
$\operatorname{Red}_{2}$ & 2.7 & $35.0,31.0$ & $40.8,24.1$ & $22.1,19.6$ & $0.60,0.38$ \\
$\operatorname{Red}_{3}$ & 2.7 & $31.0,29.2$ & $36.2,21.8$ & $21.7,20.4$ & $0.62,0.37$ \\
$\operatorname{Red}_{4}$ & 2.6 & $28.0,26.4$ & $33.3,19.9$ & $20.8,19.6$ & $0.63,0.36$ \\
$\operatorname{Red}_{5}$ & 2.4 & $34.0,30.5$ & $44.2,27.7$ & $23.0,20.9$ & $0.62,0.37$ \\
$\operatorname{Red}_{6}$ & 2.4 & $34.0,31.2$ & $43.7,27.7$ & $23.3,21.3$ & $0.62,0.37$ \\
$\operatorname{Red}_{7}$ & 2.4 & $36.0,33.1$ & $46.1,39.7$ & $24.5,22.4$ & $0.62,0.37$ \\
$\operatorname{Red}_{8}$ & 2.4 & $35.0,31.3$ & $45.2,28.1$ & $23.8,21.4$, & $0.62,0.37$
\end{tabular}

${ }^{a}$ Devices in this work. ${ }^{b}$ Voltages at $0.2 \mathrm{~mA} \mathrm{~cm}{ }^{-2} \cdot{ }^{c}$ Efficiencies of the maximum and at a brightness of $1000 \mathrm{~cd} \mathrm{~m}^{-2} \cdot{ }^{d} \mathrm{CIE}$ coordinates measured at $5 \mathrm{~mA} \mathrm{~cm}{ }^{-2}$.

a rigid molecular structure. Additionally, though they have the same HOMO levels, the LUMO level of DEX $(-2.2 \mathrm{eV})$ is higher than that of TCTA $(-2.4 \mathrm{eV})$, which means that electrons are blocked more effectively to recombine with holes for generating excitons and then emitting light in the DEX based exciplex system. Particularly, it should be mentioned that the high EQE of $11.2 \%$ is among the excellent efficiencies of exciplex-based OLEDs without any luminescent dopant (see Table S1, ESI $\dagger$ ). The detailed EL performances of Green ${ }_{1}$ and Green G $_{2}$ are listed in Table 2.

The highly efficient DEX:PO-T2T exciplex system is expected to be employed as a co-host for improving the efficiencies of PhOLEDs. According to the above results of the DEX:PO-T2T exciplex system, we believe that the exciplex should act well as a host for red emitters. The energy-level diagram and chemical structures of each material in the exciplex-based red PhOLEDs are shown in Fig. 5a-c. ${ }^{33,34}$ Red PhOLEDs were fabricated with different electron-injection layers (EILs) corresponding to different emitting layers. The exciplex DEX:PO-T2T was first successfully used as a co-host for the red phosphorescent emitter $\operatorname{Ir}(\mathbf{M D Q})_{2}$ (acac)-based OLED with the configuration of ITO/HAT-CN $(10 \mathrm{~nm}) /$ TAPC $(30 \mathrm{~nm}) / \mathbf{D E X}(10 \mathrm{~nm}) /$ DEX:PO-T2T:Ir(MDQ) $)_{2}$ (acac) $(1: 1, X \mathrm{wt} \%, X=1,3,5$, and 7 , $20 \mathrm{~nm}) /$ PO-T2T $(50 \mathrm{~nm}) / \mathrm{Liq}(2 \mathrm{~nm}) / \mathrm{Al}(120 \mathrm{~nm}) ; X=1,3,5$, and 7 correspond to the devices $\mathrm{R}_{1}, \mathrm{R}_{2}, \mathrm{R}_{3}$, and $\mathrm{R}_{4}$. Different doping ratios of the emitter were used to investigate the EL performance of the red devices. The EL characteristics of the red devices are shown in Fig. 6a-c. These devices show low onset voltages (at $0.2 \mathrm{~mA} \mathrm{~cm}^{-2}$ ) and operating voltages (at $1000 \mathrm{~cd} \mathrm{~m}^{-2}$ ) of 2.7 and $4.5 \mathrm{~V}$, respectively (as shown in Fig. S6a, ESI $\dagger$ ). As shown in Fig. 6c, the emission peak of $\operatorname{Ir}(\mathbf{M D Q})_{2}$ (acac) is redshifted with the doping ratio increase, indicting the effect of intermolecular interactions and strong polarization. ${ }^{35}$ At a low doping concentration of $1 \mathrm{wt} \%$, the device $\operatorname{Red}_{1}$ showed red emission peaking at $604 \mathrm{~nm}$ corresponding to Commission Internationale de l'Eclairage (CIE) coordinates of $(0.54,0.42)$. With increasing the doping concentration of the emitter, decreased intermolecular distances of the emitter will cause intense intermolecular interactions, and thereby red-shifted emission can be observed in devices $\operatorname{Red}_{2}-\operatorname{Red}_{4}$. Particularly, red emission with CIE coordinates of $(0.63,0.36)$ can be 


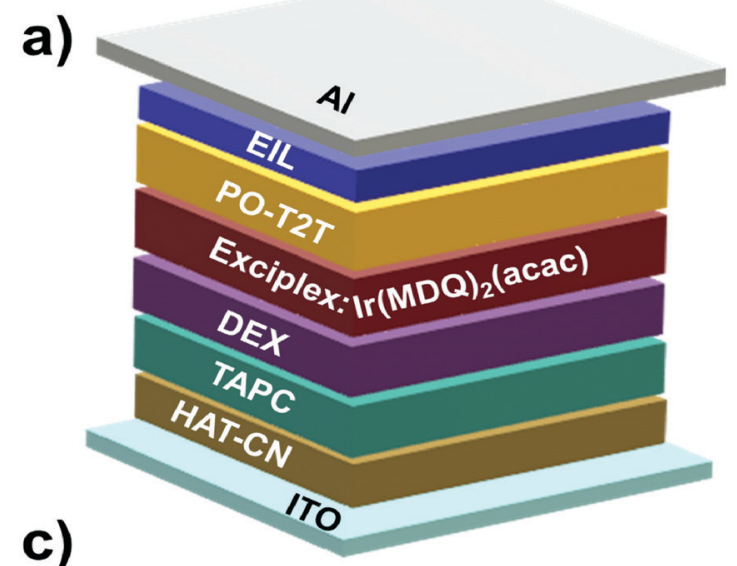

c)

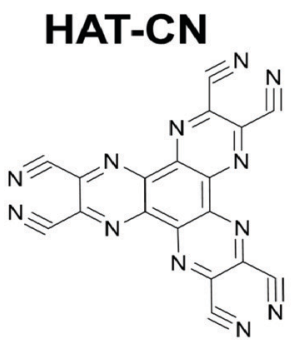

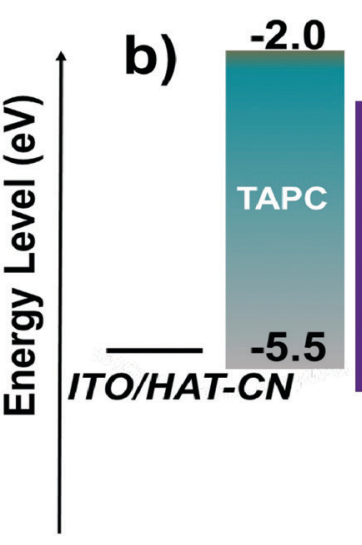

$-2.2$

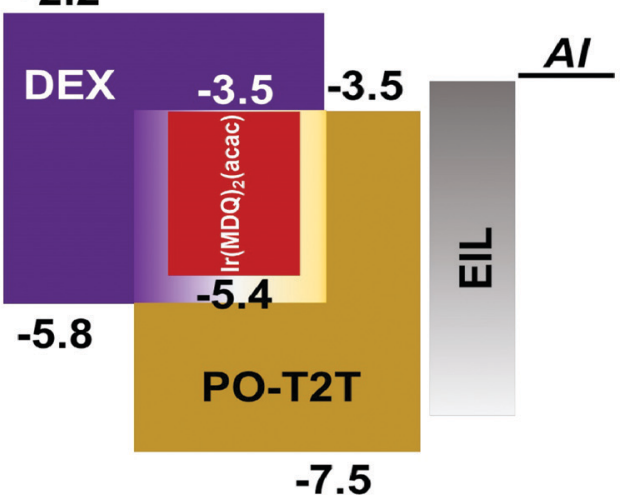

$\operatorname{Ir}(\mathrm{MDQ})_{2}($ acac $)$

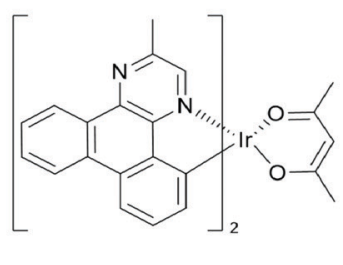

Bphen

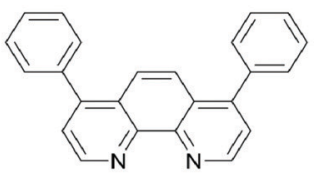

TAPC

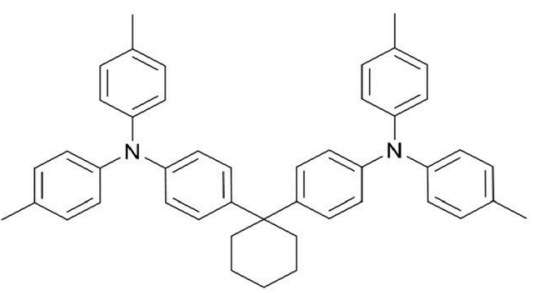

Fig. 5 (a) The optimized device structure of the red PhOLED, (b) energy-level diagram of the red PhOLED, and (c) chemical structures of the related materials in the red PhOLED.
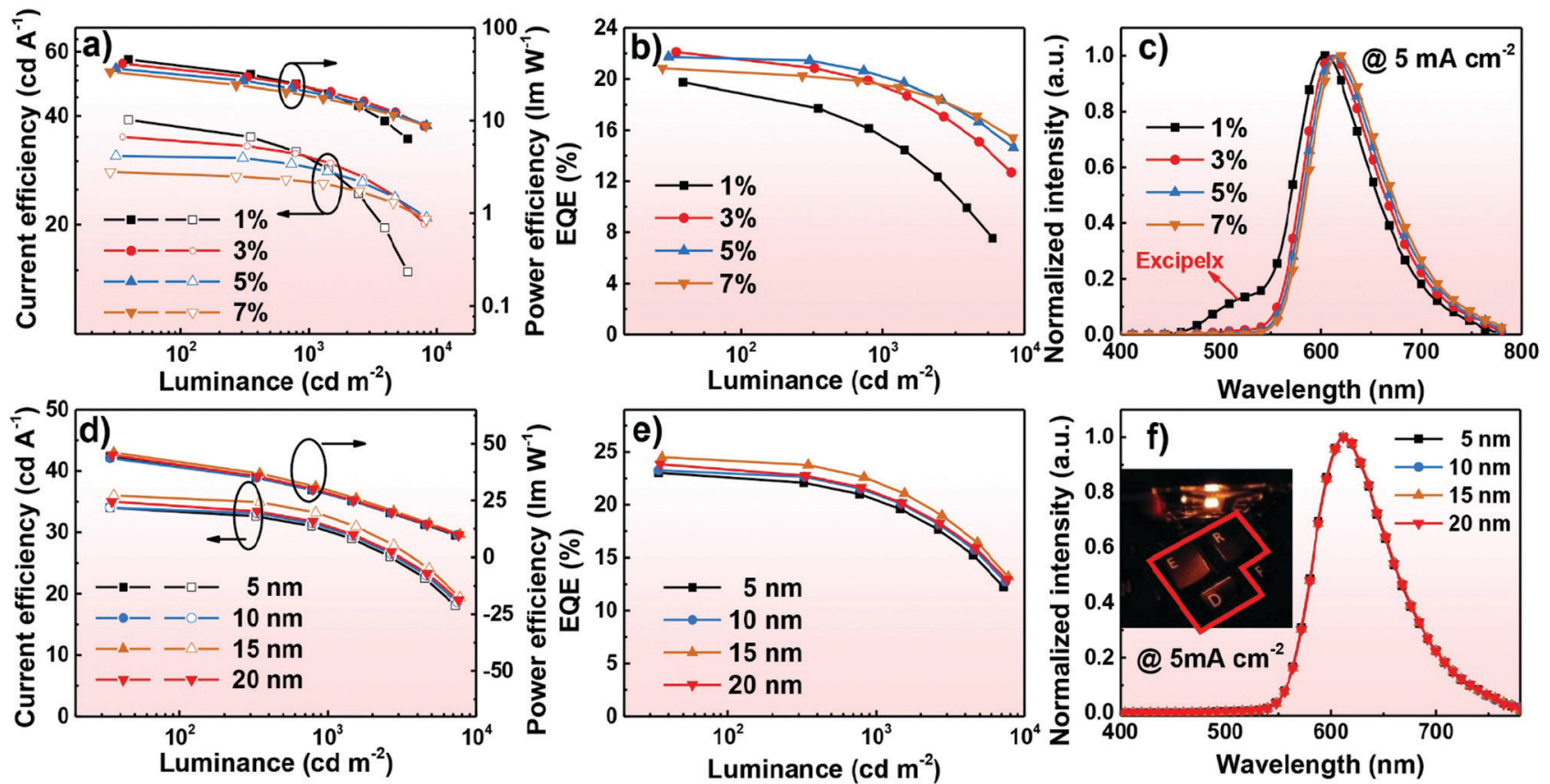

Fig. 6 (a) CE-luminance-PE plots, (b) EQE-luminance plots, and (c) EL spectra of the red OLEDs with different doping concentrations of emitters; (d) CE-luminance-PE, (e) EQE-luminance, and (f) EL spectra of the red OLEDs with LiH doped into the ETL and the inset graph is the red-emission of device $R_{7}$ at $5 \mathrm{~mA} \mathrm{~cm}^{-2}$.

achieved in device $\operatorname{Red}_{4}$ at a high doping concentration of $7 \mathrm{wt} \%$. The detailed results of device $\mathrm{R}_{1}-\mathrm{R}_{4}$ are summarized in Table 2. Notably, additional exciplex emission was observed in the $\operatorname{Red}_{1}$ device with a low doping ratio of $1 \mathrm{wt} \%$, which illustrates that the undesirable phenomenon of inadequate energy transfer was generated. ${ }^{21,27,36} \mathrm{An} \mathrm{EQE}_{\max }, \mathrm{CE}_{\max }$, and 
$\mathrm{PE}_{\max }$ of $19.7 \%, 39.0 \mathrm{~cd} \mathrm{~A}^{-1}$, and $45.3 \mathrm{~lm} \mathrm{~W}^{-1}$ were presented in the $\operatorname{Red}_{1}$ device with an emission peak of $604 \mathrm{~nm}$. With the doping ratio changes, an excellent $\mathrm{EQE}_{\max }$ of $22.1 \%, 21.7 \%$, and $20.8 \%$ was achieved for the $\mathrm{R}_{2}, \mathrm{R}_{3}$, and $\mathrm{R}_{4}$ devices, and they can maintain high EQEs of about $20 \%$ at a high brightness of $1000 \mathrm{~cd} \mathrm{~m}^{-2}$. The low efficiency roll-off is due to the sufficient utilization of excitons without energy loss and the broad recombination zone in the constructive exciplex forming co-host. It's worth noting that device $\mathrm{R}_{3}$ (doping ratio of $5 \mathrm{wt} \%$ ) can achieve desirable performance with CIE coordinates of $(0.62,0.37)$. The detailed characteristics of device $\mathrm{R}_{1-4}$ are given in Table 2 .

For further enhancing the efficiency of red OLEDs, n-doping technology was proposed in our devices. It is an effective method to enhance the electron injection and transport for decreasing the energy consumption. ${ }^{25,33}$ As a result, the $\mathrm{LiH}$ material with a $0.1 \mathrm{wt} \%$ doping ratio was doped into 4,7-diphenyl-1,10-phenanthroline (Bphen) to serve as an electroninjection layer. Hence, devices $\mathrm{R}_{5-8}$ were fabricated with structures of ITO/HAT-CN $\quad(10 \mathrm{~nm}) / \mathbf{T A P C} \quad\left(\begin{array}{llll}30 & \mathrm{~nm}\end{array}\right) / \mathbf{D E X} \quad(10 \mathrm{~nm}) /$ DEX:PO-T2T:Ir(MDQ) $)_{2}$ (acac) $(1: 1,5 \mathrm{wt} \%, 20 \mathrm{~nm}) /$ PO-T2T ( $X \mathrm{~nm}, X=5,10,15$, and 20)/Bphen: $0.1 \mathrm{wt} \% \mathrm{LiH}(50-X \mathrm{~nm}) / \mathrm{Al}$ $(120 \mathrm{~nm})$. We changed the thickness of PO-T2T to prevent the undesirable phenomenon where the lithium ions migrating into the emitting layer can seriously cause exciton quenching. ${ }^{37}$ The emitter doping ratio was fixed at $5 \mathrm{wt} \%$. From the $J-V-L$ curves displayed in Fig. S6b (ESI $\dagger$ ), the onset voltages of devices $\mathrm{R}_{5-8}$ are approximately at $2.4 \mathrm{~V}$ and low operating voltages of $3.4 \mathrm{~V}$ can be realized at a high brightness of $1000 \mathrm{~cd} \mathrm{~m}^{-2}$. These operating voltages of devices $\mathrm{R}_{5-8}$ are all lower than that of $\mathrm{R}_{1-4}$, indicting the achievement of better charge injection and transport after introducing n-doping technology. The EL characteristics of $\mathrm{R}_{5-8}$ are demonstrated in Fig. 6d-f. All devices show sole emission with a peak at $612 \mathrm{~nm}$, demonstrating that effective energy transfer can be achieved. In the end, the n-doping based devices can obtain high EQEs over 23\% and PE over $44 \mathrm{~lm} \mathrm{~W}^{-1}$. Remarkably, a high performance $\mathrm{EQE}_{\max }, \mathrm{PE}_{\max }$, and $\mathrm{CE}_{\max }$ of $24.5 \%, 46.1 \mathrm{~lm} \mathrm{~W}^{-1}$, and $36.0 \mathrm{~cd} \mathrm{~A}^{-1}$ were presented in device $\mathrm{R}_{7}$ and it can also reach a high EQE, PE, and CE of $22.4 \%$, $39.7 \mathrm{~lm} \mathrm{~W}^{-1}$, and $22.4 \mathrm{~cd} \mathrm{~A}^{-1}$ at $1000 \mathrm{~cd} \mathrm{~m}^{-2}$, so the $15 \mathrm{~nm}$ PO-T2T is effective to prevent exciton quenching. The detailed characteristics of devices $R_{5-8}$ are summarized in Table 2 . Notably, the device efficiencies of $R_{7}$ are comparable with other reported red devices employing the same dopant (see Table S2, ESI $\dagger$ ). The high EQEs and low efficiency roll-off suggest that the exciplex forming co-host using DEX has balanced charge transport and efficient utilization of excitons for radiative emission.

\section{Conclusions}

In conclusion, we fabricated an efficient green exciplex system by combining a new electron donor molecule DEX and an electron acceptor PO-T2T. Thanks to the advantages of the photophysical and electrochemical properties of DEX, the exciplex-based green OLED achieved a low operating voltage of $2.6 \mathrm{~V}$ at $100 \mathrm{~cd} \mathrm{~m}^{-2}$ as well as an excellent $\mathrm{PE}_{\max }$ and $\mathrm{EQE}_{\max }$ of $44.1 \mathrm{~lm} \mathrm{~W}^{-1}$ and $11.2 \%$. The DEX:PO-T2T exciplex as an emitter provides improved performance compared to the TCTAbased device, which is ascribed to the better carrier transport abilities and the rigid molecular structure of DEX. Furthermore, the red PhOLED employing the DEX:PO-T2T exciplex forming co-host system achieved a $\mathrm{PE}_{\max }, \mathrm{CE}_{\max }$, and $\mathrm{EQE}_{\max }$ of $46.1 \mathrm{~lm} \mathrm{~W}^{-1}, 36.0 \mathrm{~cd} \mathrm{~A}^{-1}$, and $24.5 \%$ and low operating voltages $\left(2.7 \mathrm{~V}\right.$ at $100 \mathrm{~cd} \mathrm{~m}^{-2}$ and $3.4 \mathrm{~V}$ at $\left.1000 \mathrm{~cd} \mathrm{~m}^{-2}\right)$. Therefore, it is believed that the proposed new electron donor DEX is beneficial to design an efficient exciplex system, which provides a reliable way to develop highly efficient OLEDs.

\section{Experimental}

\section{Material synthesis}

4,4,8,8,12,12-Hexaphenyl-8,12-dihydro-4H-benzo[9,1]quinolizino[3,4,5,6,7-defg]acridine. To a solution of bromobenzene (3.52 $\mathrm{g}, 22.6 \mathrm{mmol})$ in dry diethyl ether $(30 \mathrm{ml})$ was added slowly 2.4 M n-BuLi (11.3 mL, $27.1 \mathrm{mmol})$ at $-10{ }^{\circ} \mathrm{C}$ (ice-salt bath). The obtained mixture was treated by stirring at $-10{ }^{\circ} \mathrm{C}$ for $1 \mathrm{~h}$, and then a solution of trimethyl $2,2^{\prime}, 2^{\prime \prime}$-nitrilotribenzoate $(0.90 \mathrm{~g}, 2.15 \mathrm{mmol})$ in $20 \mathrm{~mL}$ dry THF was added dropwise. Then, the mixture was warmed to room temperature and stirred overnight. The reaction mixture was quenched with minimum water, and the solvent was evaporated. The crude product was treated by using boiling ethanol and filtered after cooling. The residue was cleaned with water, ethanol and hexane, and then dried under a vacuum. The dried solid was dissolved in boiling acetic acid $(30 \mathrm{~mL})$, and concentrated $\mathrm{HCl}(\mathrm{aq})(3 \mathrm{~mL})$ was added dropwise. After refluxing for 3 hours, the mixture was treated with ice water $(200 \mathrm{~mL})$. The precipitate was obtained and washed twice with ethanol, and then purified by recrystallization from ethanol/chloroform to afford the product as white crystals (1.33 g, yield: $84 \%) .{ }^{1} \mathrm{H}$ NMR (600 $\left.\mathrm{MHz}, \mathrm{CDCl}_{3}\right) \delta(\mathrm{ppm})$ : $7.10(\mathrm{t}, J=7.2 \mathrm{~Hz}, 6 \mathrm{H}), 7.04(\mathrm{t}, J=7.5 \mathrm{~Hz}, 12 \mathrm{H}), 6.98(\mathrm{t}, J=7.5 \mathrm{~Hz}$, $3 \mathrm{H}), 6.85(\mathrm{~d}, J=7.8 \mathrm{~Hz}, 6 \mathrm{H}), 6.77(\mathrm{~d}, J=7.8 \mathrm{~Hz}, 12 \mathrm{H}) .{ }^{13} \mathrm{C} \mathrm{NMR}$ $\left(\mathrm{CDCl}_{3}\right) \delta(\mathrm{ppm}): 146.26,135.44,130.25,128.58,128.50,127.53$, 126.09, 122.54, 55.90. MS (MALDI-TOF) $m / z: 739.30[\mathrm{M}+]$.

\section{General methods}

All chemicals and reagents employed in our work were obtained from commercial resources without further purification. In the synthetic routes, tetrahydrofuran (THF) and dichloromethane $\left(\mathrm{CH}_{2} \mathrm{Cl}_{2}\right)$ were purified by a PURE SOLV (Innovative Technology) purification system. ${ }^{1} \mathrm{H}$ NMR and ${ }^{13} \mathrm{C}$ NMR spectra were measured with a Bruker 600 spectrometer at room temperature. UV-vis absorption spectra were realized through a PerkinElmer Lambda 750 spectrophotometer. Photoluminescence (PL) spectra and phosphorescent spectra were measured on a Hitachi F-4600 fluorescence spectrophotometer. Differential scanning calorimetry (DSC) was performed on a TA DSC 2010 unit at a heating rate of $10{ }^{\circ} \mathrm{C} \min ^{-1}$ under nitrogen. Thermogravimetric analysis (TGA) was performed by using a TA SDT 2960 instrument with a heating rate of $10{ }^{\circ} \mathrm{C} \mathrm{min}^{-1}$ under nitrogen; the temperature at $5 \%$ weight loss was used as the decomposition temperature $\left(T_{\mathrm{d}}\right)$. 
Cyclic voltammetry was performed on a CHI600 voltammetric analyzer connected to a three-electrode system in which a platinum working electrode, a Pt wire counter electrode, and an $\mathrm{Ag} / \mathrm{AgCl}$ reference electrode were employed. Ferrocenium-ferrocene $\left(\mathrm{Fc}^{+} / \mathrm{Fc}\right)$ was used as an internal standard. Nitrogen-bubbled $0.1 \mathrm{~mol} \mathrm{~L}^{-1}$ tetrabutylammonium hexafluorophosphate $\left(\left[\mathrm{Bu}_{4} \mathrm{~N}\right] \mathrm{PF}_{6}\right)$ dichloromethane solution served as a supporting electrolyte.

\section{Device fabrication and measurement}

The OLEDs were fabricated on indium tin oxide (ITO)-covered glass substrates (15 $\Omega$ square $^{-1}$ ). All substrates were ultrasonically cleaned with acetone, ethanol and deionized water for 15 min before treating with UV-ozone for 15 min. All devices were made in a thermal deposition chamber under a pressure of $4 \times 10^{-4} \mathrm{~Pa}$. HAT-CN and Liq were deposited with a rate of $0.2 \AA \mathrm{s}^{-1}$, and $8 \AA \mathrm{s}^{-1}$ for $\mathrm{Al}$; other organic materials were treated with a rate of $2 \AA \mathrm{s}^{-1}$. Finally, the devices were encapsulated with glass sheets at room temperature. The electroluminescence (EL) information of all devices was measured using a PR 655 photometer connected to a constant current source (Keithley 2400 SourceMeter). Transient PL decays were obtained on a time-resolved fluorescence spectrometer (PL-TCSPC) of HORIB-FM-2015.

\section{Conflicts of interest}

The authors declare no conflict of interest.

\section{Acknowledgements}

The authors acknowledge financial support from the National Natural Science Foundation of China (Grant No. 51773141, 61961160731, 21572152 and 51873139) and the National Key R\&D Program of China (Grant No. 2016YFB0400700). This project is also funded by the Collaborative Innovation Center of Suzhou Nano Science and Technology (Nano-CIC), by the Priority Academic Program Development of Jiangsu Higher Education Institutions (PAPD), and by the "111" Project of the State Administration of Foreign Experts Affairs of China.

\section{Notes and references}

1 C. W. Tang and S. A. VanSlyke, Organic electroluminescent diodes, Appl. Phys. Lett., 1987, 51, 913-915.

2 L. S. Liao, K. P. Klubek and C. W. Tang, High-efficiency tandem organic light-emitting diodes, Appl. Phys. Lett., 2003, 84, 167-169.

3 G. Schwartz, M. Pfeiffer, S. Reineke, K. Walzer and K. Leo, Harvesting triplet excitons from fluorescent blue emitters in white organic light-emitting diodes, Adv. Mater., 2007, 19, 3672-3676.

4 B. B. Li, L. Gan, X. Y. Cai, X. L. Li, Z. H. Wang, K. Gao, D. C. Chen, Y. Cao and S. J. Su, An effective strategy toward high-efficiency fluorescent OLEDs by radiative coupling of spatially separated electron-hole pairs, Adv. Mater. Interfaces, 2018, 5, 1800025.

5 C. Adachi, M. A. Baldo, M. E. Thompson and S. R. Forrest, Nearly $100 \%$ internal phosphorescence efficiency in an organic light-emitting device, J. Appl. Phys., 2001, 90, 5048-5051.

6 K. S. Yook and J. Y. Lee, Organic materials for deep blue phosphorescent organic light-emitting diodes, Adv. Mater., 2012, 24, 3169-3190.

7 H. Shin, J. H. Lee, C. K. Moon, J. S. Huh, B. Sim and J. J. Kim, Sky-blue phosphorescent OLEDs with $34.1 \%$ external quantum efficiency using a low refractive index electron transporting layer, Adv. Mater., 2016, 28, 4920-4925.

8 H. Uoyama, K. Goushi, K. Shizu, H. Nomura and C. Adachi, Highly efficient organic light-emitting diodes from delayed fluorescence, Nature, 2012, 492, 234-238.

9 Y. Liu, G. H. Xie, K. L. Wu, Z. H. Luo, T. Zhou, X. Zeng, J. Yu, S. L. Gong and C. L. Yang, Boosting reverse intersystem crossing by increasing donors in triarylboron/phenoxazine hybrids: TADF emitters for high-performance solutionprocessed OLEDs, J. Mater. Chem. C, 2016, 4, 4402-4407.

10 L. Gan, Z.-D. Xu, Z.-H. Wang, B.-B. Li, W. Li, X.-Y. Cai, K.-K. Liu, Q.-M. Liang and S. J. Su, Utilizing a spiro TADF moiety as a functional electron donor in TADF molecular design toward efficient "multichannel" reverse intersystem crossing, Adv. Funct. Mater., 2019, 29, 1808088.

11 K. Goushi, K. Yoshida, K. Sato and C. Adachi, Organic lightemitting diodes employing efficient reverse intersystem crossing for triplet-to-singlet state conversion, Nat. Photonics, 2012, 6, 253-258.

12 H. Shin, S. Lee, K. H. Kim, C. K. Moon, S. J. Yoo, J. H. Lee and J. J. Kim, Blue phosphorescent organic light-emitting diodes using an exciplex forming co-host with the external quantum efficiency of theoretical limit, Adv. Mater., 2014, 26, 4730-4734.

13 J. Li, H. Nomura, H. Miyazaki and C. Adachi, Highly efficient exciplex organic light-emitting diodes incorporating a heptazine derivative as an electron acceptor, Chem. Commun., 2014, 50, 6174-6176.

14 K. H. Kim, S. J. Yoo and J. J. Kim, Boosting triplet harvest by reducing nonradiative transition of exciplex toward fluorescent organic light-emitting diodes with 100\% internal quantum efficiency, Chem. Mater., 2016, 28, 1936-1941.

15 J. H. Lee, S. H. Cheng, S. J. Yoo, H. Shin, J. H. Chang, C. I. Wu, K. T. Wong and J. J. Kim, An Exciplex Forming Host for Highly Efficient Blue Organic Light Emitting Diodes with Low Driving Voltage, Adv. Funct. Mater., 2015, 25, 361-366.

16 W. Liu, J. X. Chen, C. J. Zheng, K. Wang, D. Y. Chen, F. Li, Y. P. Dong, C. S. Lee, X. M. Ou and X. H. Zhang, Novel strategy to develop exciplex emitters for high-performance OLEDs by employing thermally activated delayed fluorescence materials, Adv. Funct. Mater., 2016, 26, 2002-2008.

17 X. K. Liu, Z. Chen, C. J. Zheng, M. Chen, W. Liu, X. H. Zhang and C. S. Lee, Nearly $100 \%$ triplet harvesting in conventional fluorescent dopant-based organic light-emitting 
devices through energy transfer from exciplex, Adv. Mater., 2015, 27, 2025-2030.

18 W. Y. Hung, P. Y. Chiang, S. W. Lin, W. C. Tang, Y. T. Chen, S. H. Liu, P. T. Chou, Y. T. Hung and K. T. Wong, Balance the carrier mobility to achieve high performance exciplex OLED using a triazine-based acceptor, ACS Appl. Mater. Interfaces, 2016, 8, 4811-4818.

19 B. Liang, J. Wang, Z. Cheng, J. Wei and Y. Wang, Exciplexbased electroluminescence: over $21 \%$ external quantum efficiency and approaching $100 \mathrm{~lm} \mathrm{~W}^{-1}$ power efficiency, J. Phys. Chem. Lett., 2019, 10, 2811-2816.

20 J. Hu, C. Y. Zhao, T. M. Zhang, X. P. Zhang, X. D. Cao, Q. J. Wu, Y. H. Chen, D. Zhang, Y. T. Tao and W. Huang, Isomeric N-linked benzoimidazole containing new electron acceptors for exciplex forming hosts in highly efficient blue phosphorescent OLEDs, Adv. Opt. Mater., 2017, 5, 1700036.

21 H. G. Kim, K. H. Kim, C. K. Moon and J. J. Kim, Harnessing triplet excited states by fluorescent dopant utilizing codoped phosphorescent dopant in exciplex host for efficient fluorescent organic light emitting diodes, Adv. Opt. Mater., 2017, 5, 1600749.

22 W. Song, I. Lee and J. Y. Lee, Host engineering for high quantum efficiency blue and white fluorescent organic light-emitting diodes, Adv. Mater., 2015, 27, 4358-4363.

23 X. K. Liu, Z. Chen, C. J. Zheng, C. L. Liu, C. S. Lee, F. Li, X. M. Ou and X. H. Zhang, Prediction and design of efficient exciplex emitters for high-efficiency, thermally activated delayed-fluorescence organic light-emitting diodes, Adv. Mater., 2015, 27, 2378-2383.

24 V. Jankus, P. Data, D. Graves, C. McGuinness, J. Santos, M. R. Bryce, F. B. Dias and A. P. Monkman, Highly efficient TADF OLEDs: how the emitter-host interaction controls both the excited state species and electrical properties of the devices to achieve near $100 \%$ triplet harvesting and high efficiency, Adv. Funct. Mater., 2014, 24, 6178-6186.

25 Q. S. Tian, L. Zhang, Y. Hu, S. Yuan, Q. Wang and L. S. Liao, High-performance white organic light-emitting diodes with simplified structure incorporating novel exciplex-forming host, ACS Appl. Mater. Interfaces, 2018, 10, 39116-39123.

26 S. F. Wu, S. H. Li, Y. K. Wang, C. C. Huang, Q. Sun, J. J. Liang, L. S. Liao and M. K. Fung, White organic LED with a luminous efficacy exceeding $100 \mathrm{~lm} \mathrm{~W}^{-1}$ without light out-coupling enhancement techniques, Adv. Funct. Mater., 2017, 27, 1701314.

27 Z. B. Wu, L. Yu, F. C. Zhao, X. F. Qiao, J. S. Chen, F. Ni, C. L. Yang, T. Ahamad, S. M. Alshehri and D. G. Ma, Precise exciton allocation for highly efficient white organic lightemitting diodes with low efficiency roll-off based on blue thermally activated delayed fluorescent exciplex emission, Adv. Opt. Mater., 2017, 5, 1700415.

28 X. K. Liu, W. Chen, H. Thachoth Chandran, J. Qing, Z. Chen, X. H. Zhang and C. S. Lee, High-performance, simplified fluorescence and phosphorescence hybrid white organic light-emitting devices allowing complete triplet harvesting, ACS Appl. Mater. Interfaces, 2016, 8, 26135-26142.

29 S. J. Su, E. Gonmori, H. Sasabe and J. Kido, Highly Efficient Organic Blue-and White-Light-Emitting Devices Having a Carrier- and Exciton-Confining Structure for Reduced Efficiency Roll-Off, Adv. Mater., 2008, 20, 4189-4194.

30 K. Udagawa, H. Sasabe, F. Igarashi and J. Kido, Simultaneous realization of high EQE of $30 \%$, low drive voltage, and low efficiency roll-off at high brightness in blue phosphorescent OLEDs, Adv. Opt. Mater., 2016, 4, 86-90.

31 X. K. Liu, Z. Chen, J. Qing, W. J. Zhang, B. Wu, H. L. Tam, F. Zhu, X. H. Zhang and C. S. Lee, Remanagement of singlet and triplet Excitons in single-emissive-layer hybrid white organic light-emitting devices using thermally activated delayed fluorescent blue exciplex, Adv. Mater., 2015, 27, 7079-7085.

32 L. Zhang, Y. X. Zhang, Y. Hu, X. B. Shi, Z. Q. Jiang, Z. K. Wang and L. S. Liao, Highly efficient blue phosphorescent organic light-emitting diodes employing a host material with small bandgap, ACS Appl. Mater. Interfaces, 2016, 8, 16186-16191.

33 Z. Bin, G. Dong, P. Wei, Z. Liu, D. Zhang, R. Su, Y. Qiu and L. Duan, Making silver a stronger n-dopant than cesium via in situ coordination reaction for organic electronics, Nat. Commun., 2019, 10, 866.

34 X. Tang, X. Y. Liu, Z. Q. Jiang and L. S. Liao, High-quality white organic light-emitting diodes composed of binary emitters with color rendering index exceeding 80 by utilizing color remedy strategy, Adv. Funct. Mater., 2019, 29, 1807541.

35 Y. J. Cho, S. K. Jeon and J. Y. Lee, Molecular engineering of high efficiency and long lifetime blue thermally activated delayed fluorescent emitters for vacuum and solution processed organic light-emitting diodes, Adv. Opt. Mater., 2016, 4, 688-693.

36 D. Y. Zhou, S. H. Zamani, Q. Wang, L. S. Liao and H. Aziz, Host to guest energy transfer mechanism in phosphorescent and fluorescent organic light-emitting devices utilizing exciplex-forming hosts, J. Phys. Chem. C, 2014, 118, 24006-24012.

37 J. H. Lee, M. H. Wu, C. C. Chao, H. L. Chen and M. K. Leung, High efficiency and long lifetime OLED based on a metaldoped electron transport layer, Chem. Phys. Lett., 2005, 416, 234-237. 